\title{
Avaliação da gestão municipal da saúde bucal na Atenção Básica: precisão do instrumento de pesquisa
}

\author{
Assessment of municipal management of oral health \\ in primary care: data collection instrument accuracy
}

Diego Anselmi Pires ${ }^{1}$

Claudia Flemming Colussi ${ }^{1}$

Maria Cristina Marino Calvo ${ }^{1}$

${ }^{1}$ Centro de Ciências da Saúde, Universidade Federal de Santa Catarina. Campus Universitário, Trindade. 88040-970 Florianopolis SC Brasil.

diegoapsc@hotmail.com

\begin{abstract}
This validation study seeks to check the accuracy of an evaluation model. In an evaluation, it is necessary to validate the precision and reliability of the data collection instrument. In this study, the Management Assessment of Oral Health in Primary Care in Santa Catarina was used as a benchmark to calculate the indicators. Its model analyzes primary data, collected via an electronic form, and secondary data, available in the Unified Health System (SUS) database. For this study, the form was applied in the cities of Santa Catarina's Coal Region at two different moments to check its reproducibility, followed by a discussion over the answers with the researcher. The results obtained were analyzed and debated in a consensus workshop with specialists in the field, detecting inaccuracies relating to the concept, the source used and the profile of the respondents themselves. The gross agreement rate in the two data collections was $87 \%$, and the inaccuracies amounted to $36 \%$ of the answers. Preferential source suggestions, question modifications and guidelines for the correct filling out of the form were some of the proposed changes, improving the original matrix and the data collection instrument.
\end{abstract}

Key words Health assessment, Oral health, Validation study, Accuracy
Resumo Este estudo de validação visa conferir a precisão de um modelo de avaliação. Em uma avaliação, existe a necessidade de validar os instrumentos de coleta de dados utilizados quanto à sua reprodutibilidade e precisão. Neste estudo, foi utilizada a Avaliação da Gestão da Saúde Bucal na Atenção Básica em Santa Catarina como referência, cujo modelo utiliza dados primários, coletados via formulário eletrônico, e secundários, disponíveis nos sistemas de informação do SUS, para cálculo dos indicadores. Para a realização deste estudo, o formulário foi aplicado nos municípios da Região Carbonífera de Santa Catarina em dois momentos distintos para verificar sua reprodutibilidade, seguidos de discussão das respostas com o pesquisador. Os resultados observados foram consolidados e debatidos em oficina de consenso com especialistas na área, detectando imprecisões relativas ao conceito, à fonte utilizada e ao perfil dos próprios respondentes. A taxa de concordância bruta observada para as duas coletas foi de 87\%, e as imprecisões somaram 36\% das respostas. Sugestões de fontes preferenciais, modificações de enunciado e orientações para o correto preenchimento do formulário foram algumas mudanças propostas, aperfeiçoando a matriz original e seu instrumento de coleta de dados.

Palavras-chave Avaliação em saúde, Saúde bucal, Estudo de validação, Precisão 


\section{Introdução}

A utilização de padrões internacionais para a apreciação da qualidade dos estudos avaliativos, ou meta-avaliação, é um processo que busca aprimorar seu desenvolvimento ou conferir credibilidade aos resultados finais de avaliações ${ }^{1}$.

Uma das categorias utilizadas como padrão de referência para julgamento de avaliações e critérios de meta-avaliação é a precisão, pois indica se a avaliação revela informações tecnicamente adequadas sobre as características da valia ou mérito do objeto ${ }^{2}$. Para a realização de uma meta -avaliação, mesmo com divergências semânticas entre autores, dois critérios são unânimes e considerados essenciais para a aceitação de um bom instrumento de coleta de dados: a confiabilidade e a validade ${ }^{3}$.

Confiabilidade é a capacidade em reproduzir um resultado de forma consistente no tempo e no espaço, ou com observadores diferentes, conferindo estabilidade, homogeneidade e equivalência entre diferentes observadores ${ }^{4}$. A confiabilidade, também compreendida por reprodutibilidade, pode ser entendida como a constância de dados e informações coletados pelo instrumento em questão. No caso de resultados diferentes, a reprodutibilidade merece ser analisada sob a ótica das diferenças reais nos dados coletados ou em variações devido a erros de mensuração ou coleta. A validade pode ser compreendida como o grau em que um instrumento realmente mede aquilo que foi concebido para medir, isto é, verifica se o instrumento retrata com precisão o fenômeno a ser estudado.

O objetivo deste estudo é conferir a precisão do instrumento de coleta de dados primários de um modelo de avaliação. O Modelo de Avaliação da Saúde Bucal na Atenção Básica de Santa Catarina ${ }^{5}$ foi utilizado como caso do estudo. Tal modelo foi construído com base nos princípios do Sistema Único de Saúde (SUS), apresentando duas dimensões para avaliar o desempenho dos municípios na formulação e execução de políticas públicas que contemplem esses princípios. A dimensão Gestão da Saúde Bucal, com foco na equidade ou garantia de acesso, tem como subdimensões a "Atuação Intersetorial", a "Participação Popular", os "Recursos Humanos" e a "Infraestrutura”. A dimensão Provimento da Atenção Básica em Saúde Bucal prioriza a integralidade e a universalidade em duas subdimensões, a "Promoção e Prevenção" e o "Diagnóstico e Tratamento", considerando o ciclo vital - crianças, adolescentes, adultos e idosos. A matriz avaliati- va prevê a coleta de dados dos 293 municípios catarinenses: os secundários nos sistemas de informação disponíveis; os primários por meio de preenchimento de formulário eletrônico. Esta avaliação vem sendo realizada periodicamente, de acordo com deliberação da Comissão Intergestores Bipartite do Estado de Santa Catarina.

Em estudos semelhantes, a utilização de dados secundários deve prever a realização de testes de consistência, com correções de valores de acordo com o preconizado para séries históricas de dados. Já para os dados primários, estudos de validação devem ser previstos para não haver dúvidas quanto ao entendimento homogêneo dos questionamentos, bem como quanto à precisão das respostas encontradas. Para a institucionalização da avaliação, é essencial considerar a precisão do modelo proposto, sua reprodutibilidade e sua importância para subsidiar o planejamento e a tomada de decisão.

\section{Metodologia}

Este artigo trata de uma pesquisa do tipo metodológica, que propõe a inquirição de métodos e procedimentos adotados como científicos, verificando a reprodutibilidade e a validade de um instrumento de coleta de dados de uma avaliação, determinando sua precisão ${ }^{6}$.

A primeira etapa foi de análise documental de relatórios, páginas de divulgação e artigos publicados sobre a avaliação da gestão da saúde bucal na atenção básica. Após, foram selecionados os municípios que participariam do estudo: $11 \mathrm{da}$ Região Carbonífera de Santa Catarina, localizada na região sul do estado, abrangendo um total de 390.756 habitantes 7 . O percurso metodológico da pesquisa está ilustrado na Figura 1.

Para determinar a confiabilidade do instrumento de coleta de dados, foi utilizada a técnica do teste-reteste. Nesta situação, o instrumento é aplicado duas vezes a um mesmo grupo de pessoas, com um intervalo de tempo definido entre as aplicações, evitando uma subavaliação em períodos de tempo muito longos e uma superavaliação, no caso de intervalos muito curtos ${ }^{3}$.

Na primeira aplicação, foi entregue uma cópia impressa do formulário de coleta de dados (Quadro 1) para o profissional designado pela gestão municipal para responder à pesquisa. Os formulários dessa etapa foram recolhidos trinta dias após a entrega, seguindo-se a segunda aplicação do mesmo formulário, novamente recolhido trinta dias depois. Nesse momento, foi realiza- 


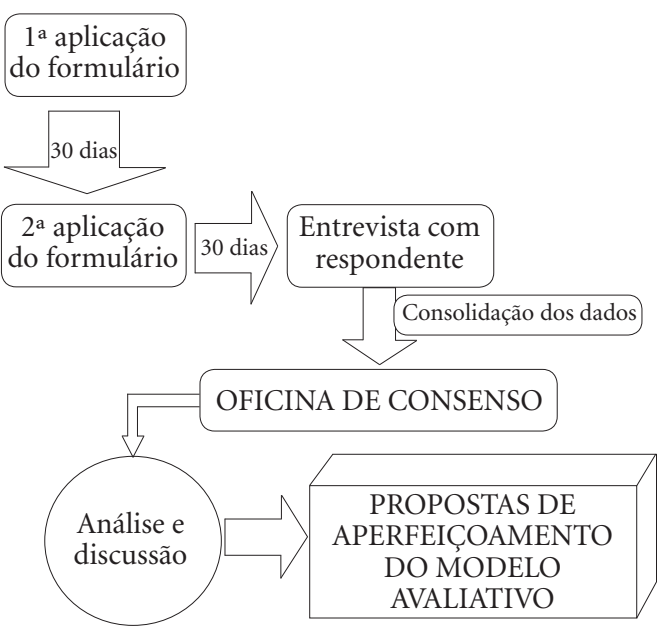

Figura 1. Percurso metodológico da pesquisa.

Fonte: autoria pessoal.

da uma entrevista com o responsável pelo preenchimento, com a finalidade de identificar facilidades, dificuldades, sugestões e, principalmente, imprecisões no preenchimento do formulário, que foram registrados em um roteiro de campo.

Dentre os três tipos de validade apontados por Contandriopoulos et al. ${ }^{4}$, a de conteúdo foi o tipo utilizado neste estudo por julgar em que proporção os itens selecionados para medir uma determinada construção teórica representam bem todas as facetas importantes do conceito a ser medido. Esta se refere à análise detalhada do conteúdo do instrumento, com objetivo de verificar se os itens propostos constituem-se numa amostra significativa do objeto em avaliação ${ }^{8}$.

Para analisar a reprodutibilidade, foram considerados os formulários de oito municípios, uma vez que um deles não aceitou participar do estudo, um não participou da primeira coleta de dados, e outro não participou por se tratar de local de atividade laboral do pesquisador. Para essa análise foi calculada a taxa de concordância bruta entre as respostas da primeira e da segunda aplicação do formulário.

As imprecisões nas respostas foram analisadas a partir das entrevistas com os nove municípios da amostra. $\mathrm{Na}$ análise das imprecisões, os resultados foram classificados em três tipos de erros identificados: E1, se o enunciado da questão não esclareceu a informação desejada ou permitiu interpretações variadas, considerou-se que a imprecisão foi no conceito; E2, quando a fonte de dados utilizada pelo entrevistado/respondente foi inadequada ou pouco-confiável, considerouse imprecisão na fonte; E3, a imprecisão estava relacionada ao entrevistado/respondente, quando este não respondeu o formulário impresso, não soube como pesquisar, não teve disposição, errou cálculos/estimativas ou falhou no registro dos dados.

Na última etapa, foi realizada uma oficina de consenso para discussão dos dados. A técnica utilizada foi o Comitê Tradicional. Foram convidados especialistas participantes das pesquisas anteriores ou atores familiarizados com a avaliação em saúde bucal para apresentação do projeto, sua matriz avaliativa, critérios e indicadores, além dos dados coletados e compilados nas etapas anteriores, sob a coordenação de um dos membros participantes $^{9-11}$.

O estudo foi aprovado pelo Comitê de Ética em Pesquisa com Seres Humanos da Universidade Federal de Santa Catarina.

\section{Resultados e discussão}

As respostas foram agrupadas de acordo com as subdimensões do Modelo de Avaliação da Gestão Municipal da Saúde Bucal na Atenção Básica. A Tabela 1 apresenta a taxa de concordância bruta das respostas nas duas coletas realizadas, segundo esses grupos.

A Tabela 2 apresenta a taxa de concordância bruta das respostas nas duas coletas realizadas para cada município e os respectivos percentuais das imprecisões, por tipo de erro encontrado.

Pela análise dos dados, fica evidente que uma alta taxa de concordância do instrumento não é garantia ou condição suficiente para conferir precisão às respostas do formulário. Um município apresentou percentual muito elevado de imprecisão nas respostas, mais de $65 \%$ equivocadas, com grande concentração de erros relacionados à fonte de dados e ao perfil do respondente. Outros sete municípios apresentaram percentuais elevados, variando de 24 a $49 \%$ de imprecisão nas respostas, com distribuição heterogênea do tipo de erro, mas com destaque para o município “ 5 ”, em que a expressiva concentração das imprecisões ocorreu relacionada ao perfil do respondente. Apenas um município apresentou um percentual de imprecisão inferior a 15\%, considerado relativamente baixo para os padrões da amostra.

Em relação à reprodutibilidade, de maneira geral, a taxa de concordância bruta do ins- 
Quadro 1. Perguntas do formulário de coleta de dados.

Perguntas de identificação:
1) Município respondente; 2) CNPJ do município;
3) Nome completo do respondente; 4) Cargo do
respondente; 5) E-mail do respondente; 6) Telefone
para contato:

\section{Perguntas da Atuação Intersetorial}

7) Número de escolas públicas (municipais e estaduais) no município que possuem cantina. 8) Número de escolas públicas (municipais e estaduais) de pré-escola e ensino fundamental no município com controle de alimentos comercializados nas cantinas em 2011.

9) Existe fluoretação da água de abastecimento público no município?

10) Qual o número total de domicílios no município em 2011?

11) Qual o número de domicílios com acesso à água tratada pela rede de abastecimento público (CASAN/SAMAE) em 2011?

12) Quanto à vigilância do teor de flúor na água de abastecimento público, o município realizou coleta mensal de amostras da água em 2011?

13) Número de escolas públicas (municipais e estaduais) de pré-escola e ensino fundamental, em 2011.

14) Número de escolas públicas (municipais e estaduais) de pré-escola e ensino fundamental com prática de escovação dental após lanches, em 2011.

\section{Perguntas da Participação Popular}

15) Número de Unidades de Saúde que atualmente disponibilizam material educativo e/ou informativo em Saúde Bucal (folder, folhetos, cartazes, painéis, etc.).

16) Na última Conferência Municipal de Saúde, houve pelo menos uma deliberação referente à Saúde Bucal?

17) Esteve presente representante da equipe técnica de saúde bucal do município na reunião de discussão e aprovação do relatório de gestão do ano de 2011?

18) O município financiou a ida de algum delegado dentista na última Conferência Estadual de Saúde?

\section{Perguntas de Recursos Humanos}

19) Quantas Unidades de Saúde interromperam atendimento odontológico devido à falta de profissional (por licença-saúde, atestado, falta ou outro motivo) por mais de dois dias consecutivos, no ano de 2011?

20) Número total de profissionais que atuavam como auxiliares fixos em odontologia em dezembro de 2011.

\section{Perguntas de Infraestrutura}

21) Em 2011, os procedimentos de endodontia foram realizados: a) No próprio município; b) em local a menos de $50 \mathrm{~km}$; c) em local a mais de $50 \mathrm{~km}$; d) Não foram realizados.

22) Número de Unidades de Saúde municipais com atendimento odontológico, incorporado a equipe de saúde da família ou não, em dezembro de 2011. 23) Número de equipamentos odontológicos utilizados para prestar atendimento em dezembro de 2011.

24) A alocação de ações e serviços de saúde bucal é realizada a partir de critério de risco da população residente?

Perguntas de Saúde Bucal em Crianças (0 a 9 anos) 25) Número de Unidades de Saúde que realizavam atividade com grupo de gestantes ou bebês com participação de algum profissional da saúde bucal (ACD, THD ou CD), em 2011.

26) Número de Unidades de Saúde que realizaram evidenciação de placa e escovação supervisionada como rotina na $1^{\text {a }}$ consulta da criança em 2011. 27) Na programação do agendamento da saúde bucal, qual percentual de consultas destinadas para criança no agendamento em 2011?

28) Número de Unidades de Saúde em que o dentista atende crianças de 0-6 anos de idade em 2011.

29) Número de Unidades de Saúde que utilizou critério de risco para priorizar atendimento às crianças nas escolas de sua área de abrangência em 2011.

\section{Perguntas de Saúde Bucal em Adolescentes} (de 10 a 19 anos)

30) Quantas Unidades de Saúde que encaminham os adolescentes de 10-19 anos de idade atendidos por outros profissionais, para orientação em saúde bucal? 31) Quantas Unidades de Saúde realizaram atividades educativas em Saúde Bucal para adolescentes, na Unidade de Saúde ou nas escolas de sua área de abrangência, em 2011?

32) Quantas Unidades de Saúde realizaram evidenciação de placa e escovação supervisionada como rotina na $1^{\text {a }}$ consulta na idade de 10-19 anos em 2011?

33) Em quantas Unidades de Saúde os pacientes de 10-19 anos foram agendados com o mesmo profissional do início ao fim do tratamento, em 2011? 34) Número total de exodontias de dentes permanentes na faixa etária de 13-19 anos: 35) Número total de procedimentos individuais na faixa etária de 13-19 anos: 
Quadro 1. continuação

\section{Perguntas de Saúde Bucal em Adultos (de 20 a 59 anos)}

36) Quantas Unidades de Saúde que possuem atualmente material educativo e/ou informativo sobre câncer bucal (folder, folhetos, cartazes, painéis, etc.)?

37) A ficha clínica odontológica possuía campo específico para anotações de exame de mucosa, em dezembro de 2011?

38) De que forma o município disponibilizou o procedimento de coleta de tecido bucal para biópsia, durante o ano de 2011? Marque as opções abaixo:a) $\mathrm{Na}$ atenção básica do município pelo dentista da Unidade de Saúde ou Estratégia Saúde da Família. b) Na atenção secundária (especializada) do município, pelo dentista do Centro de Referência em Odontologia ou outra instituição pública ou conveniada do SUS. c) Na atenção secundária (especializada) FORA do município, pelo dentista do Centro de Referência em Odontologia ou outra instituição pública ou conveniada do SUS. d) Não foi disponibilizado procedimento de coleta de tecido bucal para biópsia em 2011.

39) Número de Unidades de Saúde no município com atendimento odontológico no terceiro turno (período noturno) em dezembro de 2011. 40) Número total de exodontias de dentes permanentes na faixa etária de 20-59 anos: 41) Número total de procedimentos individuais na faixa etária de 20-59 anos:

\section{Perguntas de Saúde Bucal em Idosos} (de 60 anos ou mais)

42) Número de Unidades de Saúde que executou atividades de saúde bucal nos grupos de idosos na Unidade de Saúde ou na comunidade em 2011. 43) Quantas Unidades de Saúde realizaram atividades educativo-preventiva em Saúde Bucal e/ ou atendimento clínico em visita domiciliar para idosos com impossibilidade de locomoção até a Unidade de Saúde (acamados) no ano de 2011? 44) Quantas Unidades de Saúde possuíam sistema de atendimento ou agendamento preferencial para idosos (60 anos ou mais) em dezembro de 2011? 45) Número total de procedimentos de moldagem, adaptação e acompanhamento de prótese dentária na faixa etária acima de 60 anos:

46) Número total de exodontias de dentes permanentes na faixa etária acima de 60 anos: 47) Número total de procedimentos individuais na faixa etária acima de 60 anos:

Fonte: autoria pessoal.
Tabela 1. Percentual médio de concordância bruta para os grupos de perguntas no formulário.

$\begin{array}{cc}\text { Grupo da pergunta } & \begin{array}{c}\text { Número de } \% \text { médio de } \\ \text { perguntas } \\ \text { nogrupo }\end{array}\end{array}$

\begin{tabular}{lrl}
\hline Atuação Intersetorial & 8 & 84,38 \\
Participação Popular & 4 & 75,00 \\
Recursos Humanos & 2 & 81,25 \\
Infraestrutura & 4 & 84,31 \\
Saúde Bucal em Crianças & 5 & 82,50 \\
Saúde Bucal em Adolescentes & 6 & 93,63 \\
Saúde Bucal em Adultos & 6 & 93,58 \\
Saúde Bucal em Idosos & 6 & 91,50 \\
Total & 41 & 86,82
\end{tabular}

Fonte: autoria pessoal.

Tabela 2. Percentual de Concordância e Imprecisões - tipos de erros, segundo município.

\begin{tabular}{crrrrr}
\hline & & \multicolumn{4}{c}{ Imprecisões } \\
\cline { 3 - 6 } Município & Concordância & \multicolumn{1}{c}{ E1 } & \multicolumn{1}{c}{ E2 } & \multicolumn{1}{c}{ E3 } & E1+E2+E3 \\
\hline 1 & 85,37 & 4,88 & 14,63 & 9,76 & 29,27 \\
2 & 100,00 & 14,63 & 7,32 & 7,32 & 29,27 \\
3 & 100,00 & 4,88 & 7,32 & 12,20 & 24,39 \\
4 & 92,68 & 2,44 & 7,32 & 4,88 & 14,63 \\
5 & 87,80 & 2,44 & 2,44 & 43,90 & 48,78 \\
6 & 53,66 & 12,20 & 26,83 & 26,83 & 65,85 \\
7 & - & 17,07 & 7,32 & 12,20 & 36,59 \\
8 & 97,56 & 17,07 & 4,88 & 12,20 & 34,15 \\
9 & 78,05 & 19,51 & 7,32 & 12,20 & 39,02 \\
Total & 86,89 & 10,57 & 9,49 & 15,72 & 35,77 \\
\hline
\end{tabular}

Fonte: autoria pessoal.

trumento foi relativamente alta $(87 \%)$, mesmo quando esta taxa foi analisada por subdimensão. Apenas na subdimensão Participação Popular encontrou-se um valor um pouco menor do que nas outras, fato não relacionado ao número de perguntas de cada uma. Mesmo se analisados por pergunta, os resultados encontrados são bem favoráveis, pois cerca de $1 / 3$ das perguntas apresentou uma taxa de concordância bruta excelente, isto é, não houve discordância entre as coletas realizadas. Isto reforça a confiança na reprodutibilidade do instrumento em questão, mesmo que ainda sejam necessários alguns ajustes para sua aplicação. 
Apesar de apresentar um desempenho satisfatório em relação à reprodutibilidade do instrumento, a pesquisa observou aspectos preocupantes quanto ao número de erros encontrados nas coletas com o formulário. A Tabela 3 apresenta o percentual das imprecisões encontradas, agrupadas por área definida no modelo e pelo tipo de imprecisão identificada. De maneira geral, o valor indica o percentual do tipo de erro encontrado (E1, E2 ou E3) dentre todas as perguntas da área em questão, considerando todos os municípios selecionados.

Um índice de quase 36\% de imprecisões nas respostas, sejam relacionadas ao conceito, à fonte de dados ou ao perfil do respondente, foi encontrado na consolidação dos dados.

Todas as subdimensões do modelo avaliativo apresentaram algum tipo de imprecisão, sendo que as "Atuação Intersetorial" e "Participação Popular" apresentaram os maiores percentuais de imprecisão nas respostas às perguntas. $\mathrm{Na}$ primeira, o maior número de imprecisões estava relacionado às fontes de dados utilizadas pelos respondentes; na outra, os erros aconteceram devido a interpretações equivocadas do conceito expresso pelo indicador e por situações inerentes ao próprio respondente. As imprecisões relacionadas ao perfil do respondente também representaram o maior percentual em uma análise conjunta. As demais subdimensões apresentaram percentuais variáveis dos tipos de imprecisão, mas sempre com tendência maior para um ou outro tipo de erro.

Tabela 3. Percentual de Imprecisões segundo tipo e grupo da pergunta.

\begin{tabular}{|c|c|c|c|c|}
\hline \multirow[b]{2}{*}{ Grupo da pergunta } & \multicolumn{4}{|c|}{ Tipo de imprecisão } \\
\hline & E1 & E2 & E3 & $\begin{array}{c}\text { Total } \\
\mathrm{E} 1+\mathrm{E} 2+\mathrm{E} 3\end{array}$ \\
\hline Atuação Intersetorial & 8,33 & 37,50 & 12,50 & 58,33 \\
\hline Participação popular & 19,44 & - & 27,78 & 47,22 \\
\hline Recursos Humanos & 5,56 & 22,22 & 5,56 & 33,33 \\
\hline Infraestrutura & 11,11 & 2,78 & 16,67 & 30,56 \\
\hline Saúde Bucal em Crianças & 6,67 & 6,67 & 22,22 & 35,56 \\
\hline Saúde Bucal em Adolescentes & 9,26 & - & 14,81 & 24,07 \\
\hline Saúde Bucal em Adultos & 22,22 & - & 14,81 & 37,04 \\
\hline Saúde Bucal em Idosos & 1,85 & - & 11,11 & 12,96 \\
\hline Total & 10,57 & 9,49 & 15,72 & 35,77 \\
\hline
\end{tabular}

E1: Erro conceitual; E2: Uso de fonte inadequada; E3: Erro associado ao respondente.

Fonte: autoria pessoal.

\section{Imprecisão de Conceito (E1)}

Os problemas de imprecisão associados a erros de conceito foram detectados nos itens 7,8 , $14,15,17,20$ a 22, 26, 29 a 32, 36, 39 e 43. Para equacioná-los, foi proposto que os conceitos fossem esclarecidos no enunciado: a palavra "cantina" nos itens 7 e 8 deve ser substituída por "estabelecimento comercial com venda de alimentos para escolares na escola"; no item 14, o conceito de intersetorialidade deve estar mais claro, destacando o papel da escola na promoção da saúde bucal, sem a presença obrigatória da equipe de saúde; nos itens 15 e 41, a questão da disponibilidade, do acesso e das condições de material educativo nas Unidades Básicas de Saúde (UBS) deve estar em evidência; no item 17, a inserção da expressão “...presente nas reuniões de discussão E/OU aprovação do relatório de gestão...” facilitará o entendimento do conceito.

No item 20, sugeriu-se que fossem considerados apenas os trabalhadores contratados especificamente para exercer a função de auxiliar fixo de odontologia, Auxiliar de Saúde Bucal (ASB) ou Técnico em Saúde Bucal (TSB), e não remanejados de outra função; no item 21, existe a necessidade de evidenciar o que deve ser considerado endodontia - apenas obturação de canal radicular completada e não procedimentos gerais relacionados a esta especialidade da Odontologia, além da inserção de uma alternativa que contemple sua realização em mais de um local; já no item 22, as alterações propostas consideram o atendimento odontológico realizado na própria UBS e não referenciado para outra (acessibilidade geográfica - proximidade).

Nos itens 26 e 32, o destaque apresentado pela oficina de consenso foi que a evidenciação de placa e a escovação supervisionada devem ser associadas à prevenção individual e não atreladas à realização de procedimentos coletivos; o item 29 necessita de esclarecimentos sobre o que deve ser considerado critério de risco para atendimento às crianças; no item 30, o encaminhamento de adolescentes para Saúde Bucal, mesmo que não seja na própria UBS, deve ser contabilizado; no item 31, a definição da periodicidade destas atividades visa aumentar a sensibilidade deste indicador (valorizar quem realiza como rotina). Também deve esclarecer que as atividades educativas não se restringem à Aplicação Tópica de Flúor (ATF) e entrega de kits. No item 39, o enunciado necessita informar que o atendimento odontológico no terceiro turno deve ser considerado a partir das 19 horas; e, no item 43, a proposta de 
alteração é remover a expressão "visita domiciliar", pois pode, equivocadamente, remeter à Estratégia Saúde da Família (ESF) somente.

\section{Imprecisão na Fonte de Dados (E2)}

Os problemas de imprecisão associados a erros de seleção de fonte de dados foram detectados nos itens $7,8,10,11,13,14,19,23$ e 25 a 27 . Para solucioná-los, foi proposta a indicação das fontes preferenciais para coleta dos dados no enunciado da pergunta. Essa informação já está disponível na página eletrônica da avaliação, mas nem sempre os respondentes fazem a consulta oportuna para esse esclarecimento. Assim, para os itens 7, 8, 13 e 14, sugere-se que a informação seja obtida junto à Secretaria Municipal de Educação ou órgão semelhante; para o item 10, sugeriu-se como fonte de dados o sistema municipal que realiza a cobrança do IPTU/ITR (Imposto Predial e Territorial Urbano/Imposto Territorial Rural), pois as demais informadas pelos respondentes já estão disponíveis como dado secundário, muito embora apresentem divergências e possam estar desatualizadas. Nesse sentido, a sugestão para o item 11 é apenas reforçar a confirmação das informações com a CASAN/SAMAE ou outro órgão responsável pelo abastecimento municipal de água tratada.

Na questão 19, houve dificuldade em estabelecer uma fonte de dados oportuna e única. Trata-se de uma informação de assiduidade dos profissionais, que deve ser controlada pelos coordenadores de unidades e notificada aos responsáveis pela Saúde Bucal. Todavia, as questões pessoais e institucionais influenciam muito essa informação, tornando-a pouco precisa. Para esse item, a sugestão é a busca por outra medida para substituição. Desse modo, não houve ainda proposta de alteração para este indicador. Nos itens 23 e 25, a opção foi pela busca do dado com a Secretaria Municipal de Saúde, o que deve estar expresso no enunciado; no item 26 , sugere-se que a confirmação da informação seja realizada através de consulta aos boletins de produção ambulatorial ou ficha clínica no período em questão, o que também ocorreu para o item 27.

\section{Imprecisão relacionada ao Perfil do Respondente (E3)}

Os problemas de imprecisão associados a erros do respondente foram detectados nos itens 7 a 9,11 a 18, 20, 22 a 24, 26, 27, 29, 30, 32, 33, 36 a 39, 42 a 44 e 46. Para solucioná-los, é possível partir de uma indicação genérica de reforço ao gestor municipal quanto à importância de designar um respondente devidamente informado sobre o assunto, com orientação para recorrer às instâncias indicadas em caso de dúvida.

Como base para esta orientação geral, destacam-se os itens em que o respondente apresentou erros de conceito e de fonte de dados, ou como os itens em que o mesmo respondente utilizou fontes de dados divergentes nas duas coletas. A despreocupação com a verificação das informações também foi recorrente em vários itens, como aqueles em que o respondente informou um determinado dado na primeira coleta e descobriu a resposta correta apenas na segunda vez, ou informou valores por estimativa própria, divergentes nas duas coletas. Destaque-se que várias imprecisões referiram-se ao fato da avaliação ser realizada em um ano, mas com base nos dados do ano anterior, e houve diferença no número de unidades de saúde com atendimento odontológico implantadas em alguns municípios. Entretanto, apesar de estar bem definido no corpo do instrumento de coleta de dados, para alguns respondentes, em alguns indicadores, faltou atenção durante o preenchimento. Esta falha também se repetiu com enunciados curtos e simples, que não necessitaram de qualquer alteração.

Indesejáveis para um Cirurgião Dentista da rede de Atenção Básica ou ESF, erros básicos como o desconhecimento sobre a fluoretação ou não das águas de abastecimento, sobre critérios de risco para priorização do atendimento de crianças, sobre a idade de crianças ( 0 a 12 anos de idade e não até 15) e sobre o que é terceiro turno de atendimento também foram revelados pela pesquisa, reforçando a demanda por um instrumento com alta reprodutibilidade, preciso e em constante aperfeiçoamento, que minimize a influência dos diferentes perfis dos respondentes. Este aspecto levanta a possibilidade de que a formação curricular, em qualquer nível, ou a compreensão de habilidades gerais, competências e atribuições pertinentes a um Cirurgião Dentista atuante na rede pública seja questionada, pois conhecer o perfil deste profissional é tarefa essencial na adequação de sua formação, através inclusive de ações relacionadas à Atenção à Saúde, a gerenciamento e administração de recursos e à Educação Permanente em Saúde.

Muito embora não seja relacionado à saúde bucal, Prade ${ }^{12}$ desenvolveu e realizou um processo de validação de um instrumento de informação para gerenciamento hospitalar. Com o mesmo propósito desta pesquisa, seu estudo 
procurou fornecer subsídios para a tomada de decisões gerenciais, avaliando diferentes dimensões do escopo sistêmico da assistência hospitalar no gerenciamento deste. Vários indicadores deste instrumento não obtiveram validação e mesmo a baixa confiabilidade encontrada pode ter sido comprometida pela inaptidão do grupo de examinadores ou pela pouca prática nesse tipo de avaliação. Sugeriu que modificações fossem realizadas, visando garantir a validade deste instrumento e do sistema em questão.

Por sua vez, Stadler ${ }^{13}$, em 2011, propôs a tradução, a adaptação cultural e a validação de um questionário para avaliar o conhecimento e a conduta de médicos em relação à saúde bucal de gestantes. Estes questionários geralmente são formulados e aplicados na língua inglesa. A etapa de validação aconteceu com sua aplicação a um grupo de 36 médicos, residentes ou alunos do último ano de Medicina, em dois momentos, com intervalo de 15 dias. Para testar sua reprodutibilidade, este foi aplicado sob a forma teste-reteste, suas respostas foram analisadas quanto à coerência e concordância, encontrando-se um resultado altamente satisfatório. As alterações que se mostraram necessárias foram feitas nesta etapa. Isso reforça a necessidade de incluir, no planejamento de uma avaliação, estratégias que confiram precisão, tanto aos instrumentos utilizados quanto aos indicadores que a compõem.

Em 2010, Gonçalves et al. ${ }^{14}$, também propuseram uma tradução e validação de um instrumento consagrado na literatura internacional para averiguação das condições de saúde bucal de idosos institucionalizados. A etapa de validação com os profissionais da Enfermagem proporcionou, de maneira inovadora, uma estratégia interessante para auxiliar na compreensão dos objetivos e da utilização do instrumento. Uma teleaula, em forma de vídeo explicativo, demonstrou, de forma didática, como deveria ser efetuado o exame bucal e o preenchimento correto do instrumento. Estes profissionais declararam-se, em sua maioria, aptos a aplicar corretamente o instrumento, mas destacaram a necessidade de articular com a instituição espaços de tempo para melhor compreender sua utilização. Além disso, a incorporação gradativa no seu cotidiano de trabalho favoreceria sua melhor utilização, reforçada pelo fato de que a avaliação de indicadores de maneira isolada demonstra não ser suficiente para compreender o desenvolvimento de políticas públicas municipais ${ }^{15}$.

A utilização de metodologias participativas em todas as etapas de uma avaliação, desde a ela- boração do instrumento de coleta de dados até a análise e interpretação dos indicadores, pode ser compreendida como forma de envolver os interessados e corresponsabilizá-los nas decisões tomadas, ampliando sua utilização futura ${ }^{16}$.

Talvez essa inovação possa contribuir, significativamente, para minimizar os erros relacionados ao perfil do respondente, maior causa de imprecisões apontada por esta pesquisa. Em formato de vídeo explicativo, uma ferramenta de abrangência estadual, o Telessaúde (conferências realizadas pela internet), fruto da parceria da Secretaria de Estado da Saúde de Santa Catarina (SES-SC) e da Universidade Federal de Santa Catarina (UFSC), possibilitaria que os profissionais designados pela gestão municipal como respondentes da avaliação de saúde bucal esclarecessem dúvidas de forma interativa, em tempo real, orientando-os quanto ao entendimento e correto preenchimento do formulário eletrônico.

A partir dessas discrepâncias, todos os dados foram consolidados e subsidiaram a realização de uma oficina de consenso com especialistas da área para debater e propor alternativas para a superação destas imprecisões, bem como para ajustar o instrumento de coleta de dados, aumentando sua reprodutibilidade. A modificação de enunciados, a inserção da fonte de dados preferencial, a definição de períodos desejáveis para a informação de dados e a inclusão de explicações nos enunciados das perguntas foram algumas das modificações no instrumento propostas pelos participantes. Destaque-se que as explicações detalhadas da avaliação já ficavam disponíveis em página eletrônica devidamente identificada no corpo do formulário, mas os respondentes não indicaram ter tido acesso a estes padrões. Todas as sugestões foram encaminhadas ao setor responsável na SES-SC para utilização conveniente.

\section{Considerações finais}

Em relação à reprodutibilidade, a pesquisa evidenciou a necessidade de rever alguns aspectos importantes, pois a expectativa era que todos apresentassem as mesmas respostas nos formulários quando aplicados em momentos diferentes e respondidos pelo mesmo profissional. Este fato pode indicar diferentes compreensões acerca do conceito expresso no indicador, o uso de fontes divergentes/inadequadas ou problemas relacionados ao próprio respondente. Mesmo assim, se analisadas de acordo com as subdimensões do Modelo de Avaliação da Gestão Municipal da 
Saúde Bucal na Atenção Básica, a reprodutibilidade está em um nível bastante aceitável, acima de $86 \%$. Mesmo assim, reforça-se a necessidade de constante aperfeiçoamento do instrumento de coleta de dados.

Entretanto, ao considerar a precisão, os resultados encontrados apontam para um cuidado ainda maior com o aperfeiçoamento da matriz avaliativa. Dentre as imprecisões identificadas, foi possível perceber que a maioria destas (mais de $15 \%$ das respostas ou quase metade das imprecisões) relacionava-se ao perfil do respondente, seja porque não respondeu o formulário impresso, não soube como pesquisar, não teve disposição, errou cálculos/estimativas ou falhou no registro dos dados. Algumas imprecisões retrataram uma realidade preocupante, pois a expectativa é que estes profissionais, ocupantes de cargos de gestão da Saúde Bucal, formalizados ou não, tenham conhecimentos mínimos acerca do seu próprio trabalho, erros indesejáveis para um profissional com tamanha responsabilidade. Reiterando afirmativa anterior, este artigo não tem o intuito de questionar a veracidade das respostas, mas sim contribuir com o aperfeiçoamento de uma matriz avaliativa, verificando a precisão de seus indicadores e validando seu instrumento de coleta de dados para futuras aplicações.

Algumas imprecisões realmente estavam relacionadas ao instrumento, ao indicador, em sua essência, ou à ausência de fontes de dados preferenciais definidas no formulário eletrônico, ou mesmo a fontes inexistentes na prática institucional. A investigação conduzida faz supor que a inserção da fonte de dados preferencial em alguns indicadores pode permitir a padronização das respostas e agregar consistência às das futuras avaliações; e a mudança de enunciado, sem a necessidade de alterar o conceito ou o indicador em questão, pode evitar falhas futuras no preenchimento. Estes ajustes, sejam no próprio indicador ou no instrumento de coleta de dados, podem conferir melhor aproveitamento dos resultados de outras avaliações em saúde bucal, com dados mais fidedignos e próximos da realidade de cada gestão municipal. A correta utilização dos sistemas de informação em saúde, tanto na alimentação quanto na busca de dados, também pode potencializar o uso das avaliações das ações e serviços relacionados à saúde bucal.

A compreensão e a valorização dos conhecimentos no campo da meta-avaliação e a sua inserção no processo avaliativo, como parte do planejamento, é um passo importante para agregar confiança e permitir o aperfeiçoamento constante de um modelo avaliativo. E esse aperfeiçoamento contribuirá, consideravelmente, com o fortalecimento da saúde bucal como política pública no estado de Santa Catarina.

\section{Colaboradores}

DA Pires, CF Colussi e MCM Calvo participaram igualmente de todas as etapas de elaboração do artigo. 


\section{Referências}

1. Hartz ZMA, Felisberto E, Silva LMV. Meta-avaliação da atenção básica à saúde - teoria e prática. Rio de Janeiro: Fiocruz; 2008.

2. Worthen BR, Sanders JR, Fitzpatrick JL. Meta Avaliação. In: Worthen BR, Sanders JR, Fitzpatrick JL. Avaliação de Programas-Concepções e Práticas. São Paulo: Gente; 2004. p. 593-618.

3. Martins GA. Sobre Confiabilidade e Validade. $R G B N$ 2006; 8(20):1-12.

4. Contandriopoulos A-P, Champagne F, Potvin L, Denis JL, Boyle P. Saber preparar uma pesquisa. $3^{\mathrm{a}}$ ed. São Paulo, Rio de Janeiro: Hucitec, Abrasco; 1999.

5. Universidade Federal de Santa Catarina (UFSC). Núcleo de Extensão e Pesquisa em Avaliação em Saúde. Avaliação da Gestão da Atenção Básica em Santa Catarina. Apresenta o projeto e permite que os municípios tenham acesso aos formulários e relatórios da avaliação. [acessado 2011 jul 20]. Disponível em: http://www. nepas.ufsc.br

6. Demo P. Pesquisa e construção do conhecimento: metodologia científica no caminho de Habermas. Rio de Janeiro: Tempo Brasileiro; 1994.

7. Instituto Brasileiro de Geografia e Estatística (IBGE). Dados do Censo 2010. [acessado 2011 jul 17]. Disponível em: http://www.censo2010.ibge.gov.br/dados_ divulgados/index.php?uf $=42$

8. Hermida PMV, Araujo IEM. Elaboração e validação do instrumento de entrevista de enfermagem. Rev Bras Enferm 2006; 59(3):314-320.

9. Colussi CF, Calvo MCM. Modelo de avaliação da saúde bucal na atenção básica. Cad Saude Publica 2011; 27(9):1731-1745.

10. Colussi CF. Avaliação da qualidade da atenção em saúde bucal em Santa Catarina [tese]. Florianópolis: Universidade Federal de Santa Catarina; 2010.
11. Medina MG, Silva GAP, Aquino R, Hartz ZMA. Uso de modelos teóricos na Avaliação em Saúde: aspectos conceituais e operacionais. In: Hartz ZMA, Vieira-da-Silva L, organizadores. Avaliação em Saúde. Dos modelos teóricos à prática na avaliação de Programas e Sistemas de Saúde. Rio de Janeiro, Salvador: Fiocruz, EDIUFBA; 2005. p. 41-63.

12. Prade SS. Desenvolvimento e validação de um instrumento de informação para a assessoria do programa de controle de infecção às decisões do dirigente hospitalar [tese]. Rio de Janeiro: Fiocruz; 2002.

13. Stadler AF. Tradução e validação de um questionário de saúde bucal a médicos, obstetras e residentes em obstetrícia [dissertação]. Curitiba: Universidade Federal do Paraná; 2011.

14. Gonçalves LHT, Mello ALSF, Zimermann K. Validação de instrumento de avaliação das condições de saúde bucal de idosos institucionalizados. Esc. Anna Nery 2010; 14(4):839-847.

15. Henrique F, Calvo MCM. Grau de implantação do Programa Saúde da Família e indicadores sociais. Cienc Saude Colet 2009; 14(Supl. 1):1359-1365.

16. Costa JMBS, Felisberto E, Bezerra LCA, Cesse EAP, Samico IC. Monitoramento do desempenho da gestão da vigilância em saúde: instrumento e estratégias de uso. Cienc Saude Colet 2013; 18(5):1201-1216.

Artigo apresentado em 28/07/2013

Aprovado em 23/08/2013

Versão final apresentada em 28/08/2013 